\title{
Musical Entrainment Subsumes Bodily Gestures - Its Definition Needs a Spatiotemporal Dimension
}

\author{
MARC LEMAN \\ Ghent University, IPEM - Department of Musicology
}

\begin{abstract}
In his paper "What is entrainment? Definition and applications in musical research" (this issue), M. Clayton offers a definition of entrainment that is based on a timing dimension (relative phase relationships). However, this definition may be too limited when applied to musical entrainment. Based on the idea that human engagement with music is embodied and that gestures may condition entrainment, I suggest that the definition of entrainment be broadened so as to include a spatiotemporal dimension.
\end{abstract}

Submitted 2012 January 6; accepted 2012 July 13.

KEYWORDS: entrainment, synchronization, gestures, embodied music cognition

THE paper by Martin Clayton ("What is entrainment? Definition and applications in musical research", this issue) elucidates the notion of entrainment in a more general sense. He discusses the more distinctive features of entrainment in music and provides some examples of research about entrainment in music. Basically, I agree with the suggestion that entrainment has both an objective and subjective component. The objective component is reflected in the statement that the "evidence for entrainment will be (a) a stabilization of the relative phase relationship, and (b) the reassertion of this stability following a perturbation". He also states that "entrainment does not necessarily result in synchronization in phase between rhythms of matching periods", and that "it can involve matching periods as well as hierarchical and polyrhythmic relationships, it is out of phase as often as it is in phase; and it can fall almost anywhere on the symmetrical-asymmetrical continuum". The latter two statements fully support the idea of relative phase relationships, and they fit rather well with the idea that entrainment is a timing issue that can be measured and modeled. As for the subjective component, reference has been made to the role dynamic attention has as a contributing factor to entrainment in social interaction.

Basically, I share the viewpoint that entrainment is a highly interesting phenomenon for understanding how humans interact with music. I agree that it is possible to measure important aspects of entrainment and that case studies are important in order to get an overview of the highly diverse ways in which entrainment interferes with music making. Indeed, more case studies are needed because musical entrainment occurs at different levels and in different forms. Furthermore, I am much in favor of developing an experimental approach towards understanding musical entrainment, in addition to field observations and mathematical modeling. I also believe that the study of entrainment can further profit from approaches that focus more on subjective components, such as entrainment experiences, intentions in entrainment, and last but not least, the role of gestures and corporeal articulations in relation to entrainment. In what follows, I raise some questions about this, which illustrate the need for a broader definition of the concept of entrainment.

\section{MUSICAL ENTRAINMENT INVOLVES ACTION}

Martin Clayton seems to be aware of the need for a broader definition of entrainment when he states that metrical patterns may emerge "directly from joint action, rather than necessarily coming into existence first at the neuronal level and then being expressed behaviorally". I found this an intriguing statement, but I didn't fully grasp what Martin wanted to say. Does he mean that entrainment may be more than just a timing issue? Does he mean that human entrainment is rooted in action, in the spatial deployment of the body? Does he mean that entrainment may involve a gestural component that interacts with the time component? Unfortunately, that aspect is less well developed in the paper, although I believe that it is an essential aspect in the study of entrainment (Leman, 2007). 


\section{MUSICAL GESTURES MAY CONDITION ENTRAINMENT}

In what follows, I will concentrate on the idea that gestures may condition entrainment, and therefore, that a definition of entrainment should be broadened to include a spatiotemporal dimension that is rooted in bodily gestures. I realize that this may be considered to be a topic for further speculation. However, some of the statements that will be expressed here are supported by previous work done in the domain of empirical musicology, such as the analysis of control gestures in guqin playing in Henbing \& Leman (2007), the spatiotemporal visualization of dancing movements in Naveda \& Leman (2010), definitions and context of musical gestures in Godøy and Leman (2011), the analysis of expressiveness and intentionality in Desmet et al. (2012), the analysis of timing in Moelants et al. (2012) and the specific studies of entrainment in walking with music in Moens et al. (2010), in dancing in Van Dyck et al. (2012, in press), and gesturing, in Maes et al. (2012).

In considering the idea that gesture may condition entrainment, it may be necessary to distinguish between gross motor gesture and fine motor gestures. When people start dancing in response to music, they mainly engage in gross motor gestures. The temporal cues that are associated with these gestures can be measured and correlated with music. However, it is also possible to consider the gross motor gestures as spatiotemporal patterns (Figure 1). Such an approach may lead to a gestural topology of point clouds, a concept introduced by Naveda \& Leman (2010). Each point then shows the spatial position of the body part at a particular timing instance when it corresponds with the music. The point cloud is the result of a projection of timing instances (e.g. defining the musical meter) onto the peri-personal space - the space that the body occupies during the dance. Typically, with gross motor gestures that subsume a particular repetitive choreography, there is a large spatial region where the body part can be at a particular point in time, which ensures tolerance for synchronization variability, and therefore some flexibility in entrainment.
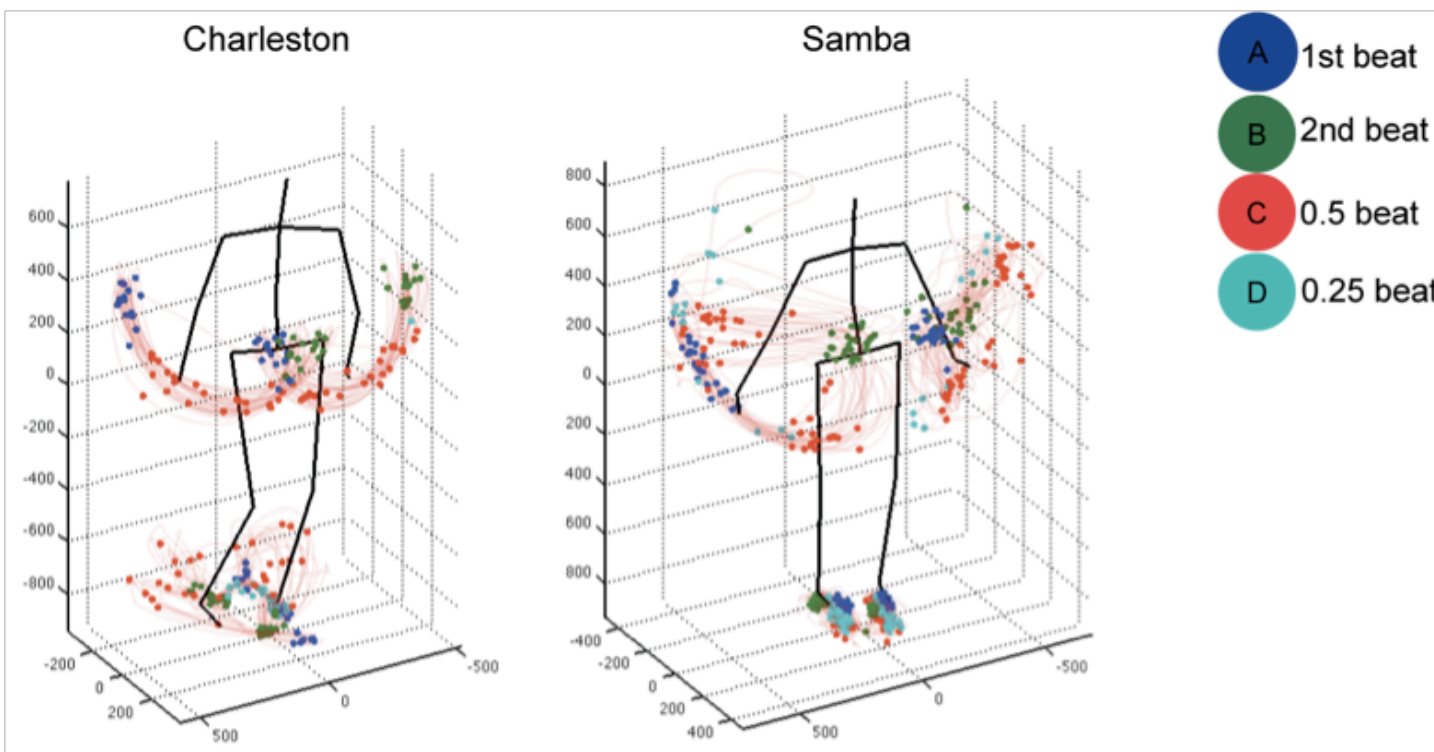

Fig. 1. Projection of metrical cues onto dancing gestures (Charleston and Samba). The metrical cues form point clouds (Naveda \& Leman, 2010).

In contrast, fine motor gestures require a more precise spatiotemporal deployment. This happens when people play music: when they sing or interact with an instrument. In that case, there is less tolerance for synchronization variability, and less flexibility in entrainment. To fully grasp my point, one needs to consider the fact that rehearsals of music ensembles often aim at getting the synchronization "right". Typically, the ensemble has to practice hard in order to quickly entrain. In this case, entrainment is not just something that happens just spontaneously. Rather, it needs careful intention, attention, and (auditory, haptic) sensing before it happens spontaneously. In other words, in order to musically entrain spontaneously, one has to practice hard, and become an experienced musician. What could be the reason for that? Again, I would argue that gestures, and the fact gestures consume time when they are spatially deployed, are of the utmost importance here.

The time fine motor gestures consumes ranges from milliseconds to a few hundreds of seconds, and their spatial resolution is measured in millimeters or smaller units at points where it really matters. Moreover, these fine-motor gestures subsume larger sequences of fine-motor gestures, having specific expressive properties (Henbing \& Leman, 2007; Desmet et al., 2012). The predictability rate of these expressive gestures is influenced by the spatial range of body parts, the inertness of the body 
parts, the inertness of the instrument, in short, factors related to physics, biomechanics, and metabolism that have to be controlled and learned. Some fine-motor gestures may subsume gross-motor gestures that serve expressive conventions (Moelants et al., 2012). Last but not least, the entire interaction is likely to involve intentional gestures that involve planning of the deployment of gestures done a few seconds ahead, and so on. In short, the musician's synchronization of fine motor patterns in view of joint activities with other musicians is far from evident. We are confronted with a complex phenomenon that involves sensorimotor control and action-perception loops, that is, embodied interaction with the environment. In my opinion, entrainment has a strong embodied component and therefore, it should be approached from a spatiotemporal perspective, supporting the idea that gestures may condition entrainment. A spatiotemporal approach to measurement is possible, as we can measure the corporeal articulation in relation to musical cues. The spatiotemporal perspective is necessary and it could clarify how a gesture exactly relates to time instances. For example, in considering the gestural details of conducting it is far from evident how to determine the exact spatial point at which subjects feel the synchronization with the beat. By using point clouds, we can locate the musical beat point with respect to the movement. However, this spatial location of the musical cue cannot necessarily be found at the same location of the perceived and enacted beat point. It may be that the synchronization, and the entrainment, is not determined by time points but by short gestural events (cf. also Maes et al., 2012).

\section{LEVELS OF EMBODIED ENTRAINMENT}

I would like to conclude my brief reflection by considering the idea that embodied entrainment should be studied at at least three different levels, namely the levels of context, gesture-repertoire, and sensorimotor control. First of all, consider the context in which the musical action happens. This may indeed affect entrainment in the sense that entrainment responses may be conditionally dependent on the environmental constraints according to which the individual acts. A good example is the intergroup entrainment where two groups try to maintain their identity by not entraining to each other. As mentioned by Martin Clayton, there are ethnographic studies that document how groups of musicians consider playing in time in relation to their identity as a group. In a pilot experiment carried out at an international summer school for systematic musicology (ISSSM 2009), two percussion groups (17 and 18 subjects) were marching in the street at a slightly different but spontaneous tempo, playing the same rhythmic pattern. When they crossed each other, they were influenced by each other's tempo and adapted their phase. When they continued marching, they quickly drifted away from each other (Figure 2 and Figure 3). Clearly, the entrainment was conditioned by gross motor gestures. Synchronization became possible and the groups were vulnerable for entrainment effects. The experiment took place on the street, between building blocks. It was found that spatial aspects, such as reflection on walls, as well as small phase shifts when two groups are approaching (due to the time that sounds need to travel), could have influenced the entrainment. Effects of physical space are known in music, but their relation to entrainment needs to be studied in more detail.
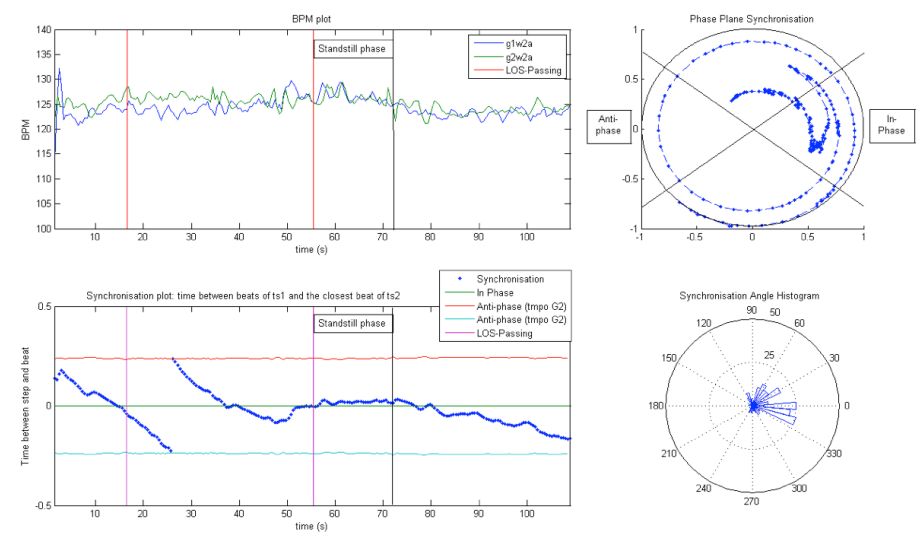

Fig. 2. Top-left: the beats per minute (BPM) over time of two groups (annotated manually from audio files). Down-right: synchronization plot of the time between the beats of the two groups. Top-right: phase plane plot. Top-right: synchronization angle histogram. See also Moens et al. (2010). 


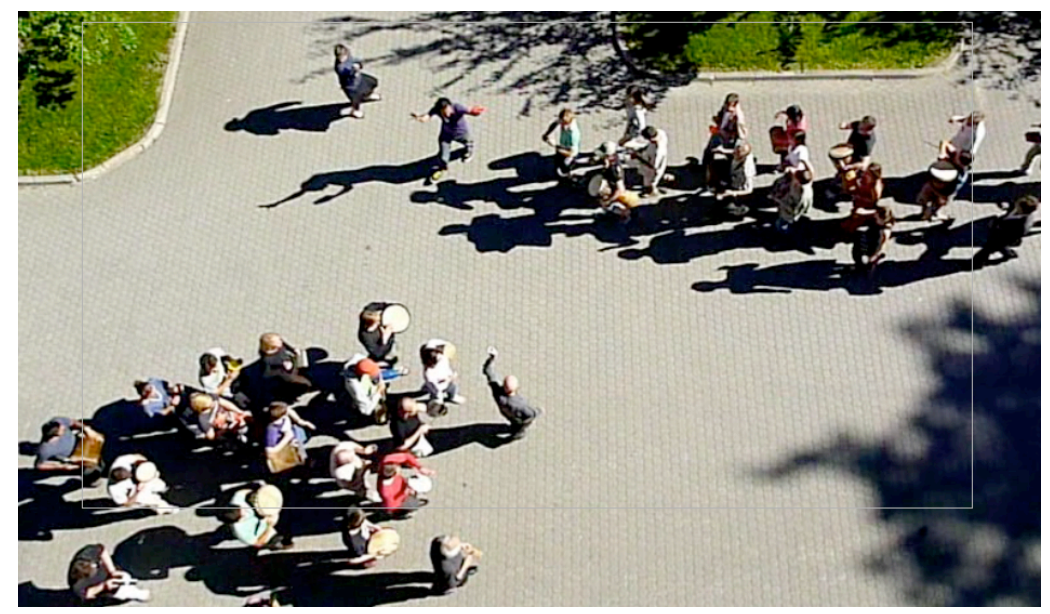

Fig. 3. Two percussion groups encounter each other (See Figure 1 at about $50 \mathrm{sec}$.)

Secondly, there is the gesture-repertoire that may affect entrainment. A gesture-repertoire is the set of gesture commands and their outcomes that is somehow stored in memory and used for the next gesture production or the next interpretation of what is sensed. As mentioned before, for musicians it may take some time to learn to develop a repertoire of expressive musical gestures with regards to exact timing. The difficulty of being entrained is related to the fact that the repertoire involves fine motor patterns that subsume larger scale expressive gestures that matter at tens of milliseconds. It is likely that the neural clocks involved are spatiotemporal clocks, in other words, that they are embodied clocks that measure time through bodily movement.

Thirdly, there are sensorimotor cycles that may affect entrainment. It suffices here to say that a large part of fine-motor musical gestures (such as lip control during wind instrument playing) is controlled by sensing, and by learned (and automated) patterns that interfere with sensing at millisecond levels. However, the control of body parts assumes time, and therefore sensing should be considered as an additional constraint for entrainment.

\section{CONCLUSION}

To sum up, my point is that the concept of entrainment, when applied to music, should take into account the fact that our interactions with music are based on our body, and therefore that corporeal movements will have an impact on entrainment. From that perspective, I would be inclined to consider musical entrainment not purely in terms of timing but rather in terms of spatiotemporal deployment of gestures. Furthermore, I believe that a distinction can be made between gross and fine motor activities and I believe that different levels should be distinguished at which entrainment can occur. However, a perspective on entrainment that focuses on timing issues, as proposed by Martin Clayton, is perfectly compatible with a perspective on entrainment that focuses on spatiotemporal issues. I consider this paper as a step towards a further spatiotemporal exploration of entrainment in music research.

\section{REFERENCES}

Desmet, F., Nijs, L., Demey, M., Lesaffre, M., Martens, J-P., \& Leman, M. (2012). Assessing a clarinet player's performer gestures in relation to locally intended musical targets. Journal of New Music Research, Vol. 4, No. 1, pp. 31-48.

Godøy, R. I., \& Leman, M. (Eds.) (2009). Musical gestures: sound, movement, and meaning. New York, NY: Routledge.

Henbing, L., \& Leman, M. (2007). A gesture-based typology of sliding-tones in guqin music. Journal of New Music Research, Vol. 36, No. 2, 61-82.

Leman, M. (2007). Embodied music cognition and mediation technology. Cambridge, MA: The MIT Press.

Maes, P.-J., Amelynck, D., \& Leman, M. (2012). Dance-the-Music. An educational platform for the modeling, recognition and audiovisual monitoring of dance steps using spatiotemporal motion 
templates. EURASIP Journal on Advantages in Signal Processing (special issue: Audiovisual Analysis and Synthesis of Body Motion for Performing Arts), Vol. 35, pp. 1-16.

Moelants, D., Demey, M., Grachten, M., Wu, Chai-Fen, \& Leman, M. (2012). The influence of an audience on performers: a comparison between rehearsal and concert using audio, video and movement data. Journal of New Music Research, Vol. 41, No. 1, 67-78.

Moens, B., van Noorden, L., \& Leman, M. (2010). D-Jogger: Syncing Music with Walking. Paper presented at the Proceedings of SMC Conference 2010, Barcelona.

Naveda, L. A., \& Leman, M. (2010). The spatiotemporal representation of dance and music gestures using topological gesture analysis (TGA). Music Perception, Vol. 28, No. 11, pp. 93-111.

Van Dyck, E., Moelants, D., Demey, M., Deweppe, A., Coussement, P., \& Leman, M. (2012, in press). The impact of the bass drum on music-induced movement. Music Perception. 